\title{
Review of Fresnel Incoherent Correlation Holography with linear and non-linear correlations
}

\author{
Vijayakumar Anand ${ }^{1, *}$, Tomas Katkus, ${ }^{1}$ Soon Hock $\mathbf{~ g g ,}{ }^{1}$ and Saulius Juodkazis ${ }^{1,2,3, * *}$ \\ ${ }^{I}$ Optical Sciences Centre and ARC Training Centre in Surface Engineering for Advanced Materials (SEAM), School of Science, Swinburne \\ University of Technology, Hawthorn, VIC 3122, Australia; \\ ${ }^{2}$ Melbourne Centre for Nanofabrication, ANFF, 151 Wellington Road, Clayton, VIC 3168, Australia. \\ ${ }^{3}$ Tokyo Tech World Research Hub Initiative (WRHI), School of Materials and Chemical Technology, Tokyo Institute of Technology, 2-12-1, \\ Ookayama, Meguro-ku, Tokyo 152-8550, Japan. \\ *Corresponding author: vanand@swin.edu.au; ** corresponding author: sjuodkazis@swin.edu.au
}

\begin{abstract}
Fresnel incoherent correlation holography (FINCH) is a well-established incoherent imaging technique. In FINCH, three selfinterference holograms are recorded with calculated phase differences between the two interfering, differently modulated object waves and projected into a complex hologram. The object is reconstructed without the twin image and bias terms by a numerical Fresnel back propagation of the complex hologram. A modified approach to implement FINCH by a single camera shot by pre-calibrating the system involving recording of the point spread function library and reconstruction by a non-linear cross-correlation has been introduced recently. The expression of the imaging characteristics from the modulation functions in original FINCH and the modified approach by pre-calibration in spatial and polarization multiplexing schemes are reviewed. The study reveals that a reconstructing function completely independent of the function of the phase mask is required for the faithful expression of the characteristics of the modulating function in the image reconstruction. In polarization multiplexing method by cross-correlation, a partial expression was observed, while in spatial multiplexing method by cross-correlation, the imaging characteristics converged towards a uniform behavior.

Keywords: Digital holographic imaging, Fresnel incoherent correlation holography, Holographic techniques, Imaging systems, Incoherent holography and Speckle noise.
\end{abstract}

Fresnel incoherent correlation holography (FINCH) is one of the well-established incoherent digital holography techniques [1, 2] developed by Prof. Joseph Rosen and Prof. Gary Brooker. In FINCH, the hologram of an object is recorded using the selfinterference principle, where both interfering waves are object waves derived from the same object but differently modulated by quadratic phase masks (QPMs) with different focal distances [35]. Three holograms are recorded in FINCH with pre-calculated phase differences between the two interfering object waves and superimposed to generate a complex hologram. The above phaseshifting approach is needed to remove the twin images and bias term present in the in-line hologram during reconstruction [6]. The three-dimensional image of the object is reconstructed by propagating the complex hologram to one of the image planes of the two QPMs [1]. In the subsequent studies, the super resolution capabilities of FINCH was revealed [7-10] and so FINCH was adapted into various methods as a resolution booster [11, 12]. FINCH underwent different developments to achieve threedimensional imaging with a single camera shot [13-19]. A micropolarizer array [13] enabled capturing four polarization multiplexed camera shots in a single shot followed by a computational interpolation which synthesized the intermediate pixel values. In [14], a checker board grating was used to spatially multiplex multiple shots in the same shot by sacrificing the field of view. In [15, 16] off-axis configuration was applied, while in [17], a geometric phase lens was used.

In the above developments, the imaging characteristics of FINCH remained unchanged as the fundamental principle of hologram formation and reconstruction remained unaltered. In a recent study [19], the first version of FINCH was studied in a new light using a single camera shot and a non-linear reconstruction method [20]. In the first step, a point object was scanned along the optical axis at all possible axial locations and the corresponding point spread holograms (PSHs) were recorded. In the next step, an object was placed between the axial boundaries of the PSHs and an object hologram was recorded. This method was inspired from coded aperture correlation holography (COACH) [3]. By this approach, a one-time calibration procedure enabled single camera shot capability in FINCH.

In general, the imaging characteristics of FINCH are affected by the phase functions used for modulating the object waves. For instance, in [21], a spiral phase plate was used instead of the QPM to modulate one of the object waves and create the hologram. This hologram when reconstructed, generated edge enhanced images of the object. However, in [19], as the hologram reconstruction was converted into a pattern recognition problem, FINCH showed a higher axial resolution which is not a property of the earlier version of FINCH. In another recent study [22], where an axicon was used instead of a QPM to generate the hologram in FINCH, the higher focal depth and lower spectral sensitivity which are characteristics of a Bessel beam were not observed during the reconstruction [22]. These surprising aspects have not been investigated yet. In this study, we review the spatial and spectral imaging characteristics of the two versions of FINCH: polarization multiplexing and spatial multiplexing schemes under reconstruction by back propagation and cross-correlation by a nonlinear filter. The two versions with polarization and spatial multiplexing are shown in Figs. 1(a) and 1(b) respectively.

The optical configuration of FINCH in polarization multiplexing scheme [8] and spatial random multiplexing scheme $[1,19,22]$ are shown in Figs. 1(a) and 1(b) respectively. In the 
polarization multiplexing scheme (Fig. 1(a)), a thick object is critically illuminated by a spatially incoherent source. The light from the object is collected by a refractive lens located at $z_{S}$ and is incident on a polariser $\mathrm{P}_{1}$ oriented at $45^{\circ}$ with respect to the active axis of the spatial light modulator (SLM) located at $z_{d}$ from the refractive lens. On the SLM, a QPM with a focal length of $z h / 2$ is displayed which modulates about half of the incident light while the remaining is unmodulated. A self-interference between the two beams is obtained by a second polariser $\mathrm{P}_{2}$ oriented at $45^{\circ}$ with respect to the active axis of the SLM, which renders both beams with the same polarization orientation. The hologram is recorded by an image sensor located at a distance of $z_{h}$ from the SLM. Three phase shifts $\theta_{k}=0,2 \Pi / 3$ and $4 \Pi / 3$ are introduced to the QPM and the corresponding holograms are recorded, projected into complex space and a complex hologram is obtained. The different planes of the object are reconstructed by propagating the complex hologram numerically by the respective distances.

For a point object with an amplitude $\sqrt{I_{o}}$ located at $Z_{\mathrm{S}}$ from the refractive lens with a focal length of $f_{1}$, the complex amplitude entering the lens is given as $C_{1} \sqrt{I_{o}} Q\left(1 / z_{S}\right)$, where $Q(b)=e^{j \frac{\pi b R^{2}}{\lambda}}$, where $R=\sqrt{x^{2}+y^{2}}, 1 / b$ is the focal distance and $C_{m}$ is a complex constant. The complex amplitude exiting the lens is given as $C_{2} \sqrt{I_{o}} Q\left(1 / z_{1}\right)$, where $z_{1}=f_{1} z_{S} /\left(z_{s}-f_{1}\right)$. The complex amplitude introduced by the SLM is given as $e^{j \Phi_{k}(x, y)}$ and $k=1$ to $n$. Assuming that $z_{d}$ is small and considering that the $45^{\circ}$ polarization orientation with respect to the active axis of SLM generates a modulated and unmodulated beam, the complex amplitude after the SLM can be approximated as $C_{3} \sqrt{I_{o}} Q(1 /$ $\left.z_{1}\right)\left(1+e^{j \Phi_{k}(x, y)}\right)$. It must be noted that the ' + ' symbol does not have any effect until the complex amplitudes pass through $\mathrm{P}_{2}$, as before $\mathrm{P}_{2}$ the two components have orthogonal polarizations and therefore cannot interfere. The self-interference point spread hologram at the sensor plane is given as $I_{P S H}(k) \simeq \mid\left\{C_{3} \sqrt{I_{o}} Q(1 /\right.$ $\left.\left.z_{1}\right)\left(1+e^{j \Phi_{k}(x, y)}\right)\right\}\left.\otimes Q\left(1 / z_{h}\right)\right|^{2}$, where ' $\otimes$ ' is a $2 \mathrm{D}$ convolutional operator. The complex hologram $H_{P S H}$ formed by the superposition of recorded phase shifted $I_{P S H S}$ is given as $H_{P S H}=I_{P S H}(k=$ 1) $\left(e^{-j \theta_{3}}-e^{-j \theta_{2}}\right)+I_{P S H}(k=2)\left(e^{-j \theta_{1}}-e^{-j \theta_{3}}\right)+I_{P S H}(k=$ 3) $\left(e^{-j \theta_{1}}-e^{-j \theta_{2}}\right)$.

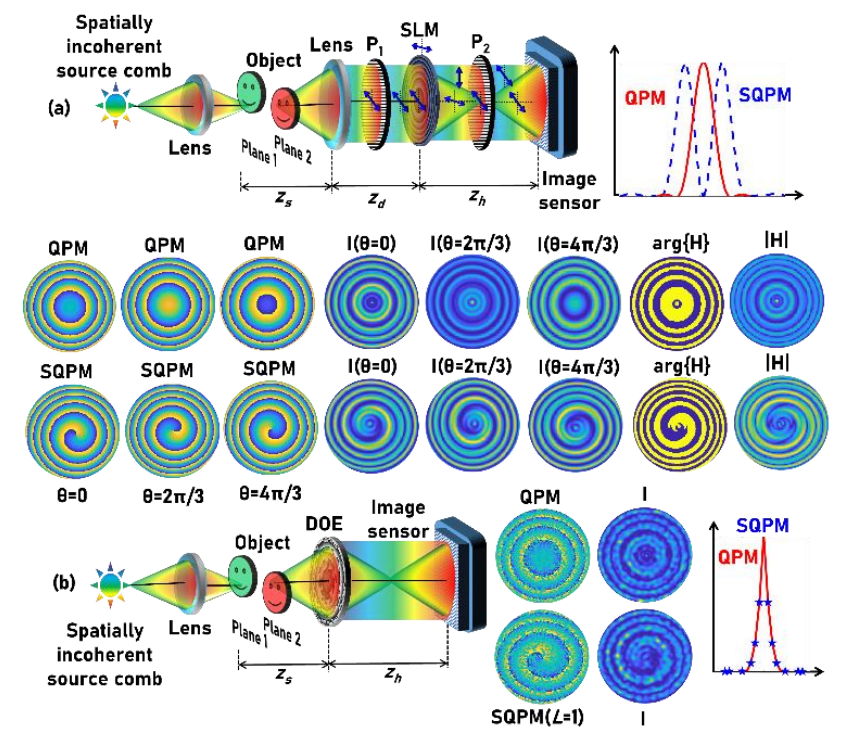

Fig. 1 Optical configuration of FINCH with (a) polarization multiplexing and (b) spatial multiplexing.
As the illumination is incoherent, a complicated object $O$ may be considered as a collection of independent point objects and the object intensity is given as $I_{O}(k)=I_{P S H}(k) \otimes O$. The object hologram is given as $H_{O}=I_{O}(k=1)\left(e^{-j \theta_{3}}-e^{-j \theta_{2}}\right)+I_{O}(k=$ 2) $\left(e^{-j \theta_{1}}-e^{-j \theta_{3}}\right)+I_{O}(k=3)\left(e^{-j \theta_{1}}-e^{-j \theta_{2}}\right)$. The image of the object is reconstructed by a back propagation given as $O^{\prime}=$ $\left|H_{O} \otimes Q\left(-1 / z_{r}\right)\right|$, where $Z_{r}$ is the reconstruction distance. The factor $\Phi_{k}(x, y)$ is the controller of the characteristics of imaging. In [1], $\Phi_{k}(x, y)=\left\{\frac{2 \pi R^{2}}{\lambda z_{h}}\right\}_{2 \pi} M+M \theta_{k}+(1-M)$, where $M$ is a binary random matrix with a scattering degree $\sigma$, while in [8], $\Phi_{k}(x, y)=\left\{\frac{2 \pi R^{2}}{\lambda z_{h}}+\theta_{k}\right\}_{2 \pi}$ and in [21], $\Phi_{k}(x, y)=\left\{\left(\frac{2 \pi R^{2}}{\lambda z_{h}}+\right.\right.$ $L \psi)\}_{2 \pi} M+M \theta_{k}+(1-M)$, where $L$ is the topological charge and $\Psi$ is the azimuthal angle. The reconstruction mechanism is independent of the beam modulations involved as the hologram is always propagated to a plane of interest by a convolution with $\mathrm{Q}(\mathrm{b})$. On one hand, this approach demands at least three camera shots and decreases the time resolution and on the other hand it enables faithful expression of the modulation function $\Phi(x, y)$ in the imaging characteristics.

In the recent studies [19, 22], a modified approach was used with a compact configuration (Fig. 1(b)) and the reconstruction was carried out by cross-correlation with a non-linear filter [20] with the PSH. The mathematical analysis of the optical configuration (Fig. 1(b)) follows next. A point object with an amplitude of $\sqrt{I_{o}}$ is considered. The complex amplitude at a distance of $z_{S}$ from the point and entering the SLM is given as $C_{1} \sqrt{I_{o}} Q\left(1 / z_{S}\right)$. The phase function displayed on the SLM is given by $\Phi(x, y)=\left\{\left(\frac{\pi R^{2}}{\lambda f_{2}}\right)\right\}_{2 \pi} M+\varphi(x, y)(1-M)$, where the first function collimates the incoming light (when $f_{2}=z_{s}$ ) while the second function can be a QPM as in [1], a spiral phase plate as in [21] or an axicon as in [22]. The complex amplitude leaving the SLM is given as $C_{4} \sqrt{I_{o}} Q\left(1 / z_{S}\right) e^{j \Phi(x, y)}$ and the self-interference point spread hologram is given as $I_{P S H}=\mid C_{4} \sqrt{I_{o}} Q(1 /$ $\left.z_{S}\right)\left.e^{j \Phi(x, y)} \otimes Q\left(1 / z_{h}\right)\right|^{2}$. The object hologram for an object $O$ is given as $I_{O}=I_{P S H} \otimes O$. The object hologram if cross-correlated with $I_{P S H}$, the object can be reconstructed as $O^{\prime}=I_{P S H} \otimes O * I_{P S H}$, where ' $*$ ' is a $2 \mathrm{D}$ cross-correlation operation. The result of this process is the image of the object formed by sampling the object by the autocorrelation function $I_{P S H} * I_{P S H}$. If the point spread hologram can be recorded, then the object can be reconstructed by the above procedure. However, a direct cross-correlation between two positive intensities will result in background noise. To eliminate this noise, a non-linear adaptive correlation filter given by

$\left|\mathcal{F}^{-1}\left\{\left|\tilde{I}_{P S H}\right|^{\alpha} \exp \left[i \arg \left(\tilde{I}_{P S H}\right)\right]\left|\tilde{I}_{O}\right|^{\beta} \exp \left[-i \arg \left(\tilde{I}_{O}\right)\right]\right\}\right| \quad$ was applied, where $\alpha$ and $B$ are tuned between -1 and 1 , to obtain the minimum entropy given as $S(p, q)=-\sum \sum \phi(m, n) \log [\phi(m, n)]$, where $\phi(m, n)=|C(m, n)| / \sum_{M} \sum_{N}|C(m, n)|, \quad(m, n)$ are the indexes of the correlation matrix, and $Q(m, n)$ is the correlation distribution.

In this approach, the reconstructing function is dependent upon the beam modulation functions. In Fig. 1(a), the point reconstructions for a QPM and a spiral QPM (SQPM) are different owing to the system independent reconstruction mechanism. On the other hand, the point reconstructions are the same for both QPM and SQPM in Fig. 1(b). In original FINCH with reconstruction by convolution with $\mathrm{Q}(1 / \mathrm{b}), \mathrm{FINCH}$ exhibited a 
lower axial resolution. In [22], a substantial increase in axial and spectral resolutions was observed which are different from the properties of the original versions of FINCH. It must be noted that even in the polarization multiplexing scheme [8], the reconstruction by cross-correlation can be applied. In this study, the spatial and spectral correlations for different functions of $\Phi(k)$ in different optical configurations are investigated. The lateral resolution of the above two versions of FINCH cannot be compared due to requirement of recording $\mathrm{PSH}$ with a pinhole in the second version as the maximum resolution is governed by the pinhole diameter which samples the object space than the system configuration.

For the simulative studies, the following design parameters are considered: $\lambda=0.6 \mu \mathrm{m}, Z_{s}=20 \mathrm{~cm}, Z_{d}=0, Z_{h}=40 \mathrm{~cm}$, aperture diameter $D=4 \mathrm{~mm}, N A=0.01$ and $z_{r}=20 \mathrm{~cm}$. Direct imaging with a lens of phase $\Phi_{k}=-\Pi R^{2} / \lambda f_{3}$ and focal length $f_{3}=\left(1 / Z_{S}+1 / Z_{h}\right)^{-1}=$ $13.33 \mathrm{~cm}$, FINCH in polarization multiplexing scheme and spatial multiplexing scheme with a QPM of phase $\Phi_{k}=\left\{-\Pi R / \lambda f_{4}+\theta_{k}\right\}$ and focal length $f_{4}=z_{b} / 2=20 \mathrm{~cm}$, an axilens [23] with a phase $\Phi_{k}=\{-$ $\left.\Pi R^{2} / \lambda f_{5}(R)+\theta_{k}\right\}$ and a focal length $f_{5}(R)=\left\{f_{0}+\left(4 \Delta y D^{2}\right) R\right\}$, where $\Delta z$ $=2 \mathrm{~cm}$ and $f_{0}=19 \mathrm{~cm}$, an axicon with a phase $\Phi_{k}=\left\{-2 \Pi / \lambda(\gamma R)+\theta_{k}\right\}$ where $Y=0.005$ radians $[24,25]$ and a SQPM $\left(\Phi_{k}=-\Pi R^{2} / \lambda f_{4}+L \Psi\right.$ $+\theta_{k}$ ) with $L=1[26,27]$ are compared. In the spatial multiplexing scheme, the scattering ratio $\sigma$ is selected as 0.5. A smiley object is used for the studies. The reconstruction is carried out using Fresnel back propagation $\left(z_{r}=20 \mathrm{~cm}\right)$, non-linear correlation $(\alpha=0$ and $B=0.5$ ) and Lucy-Richardson algorithm (LRA) [28]. The reconstruction results for the above cases are shown in Fig. 2.

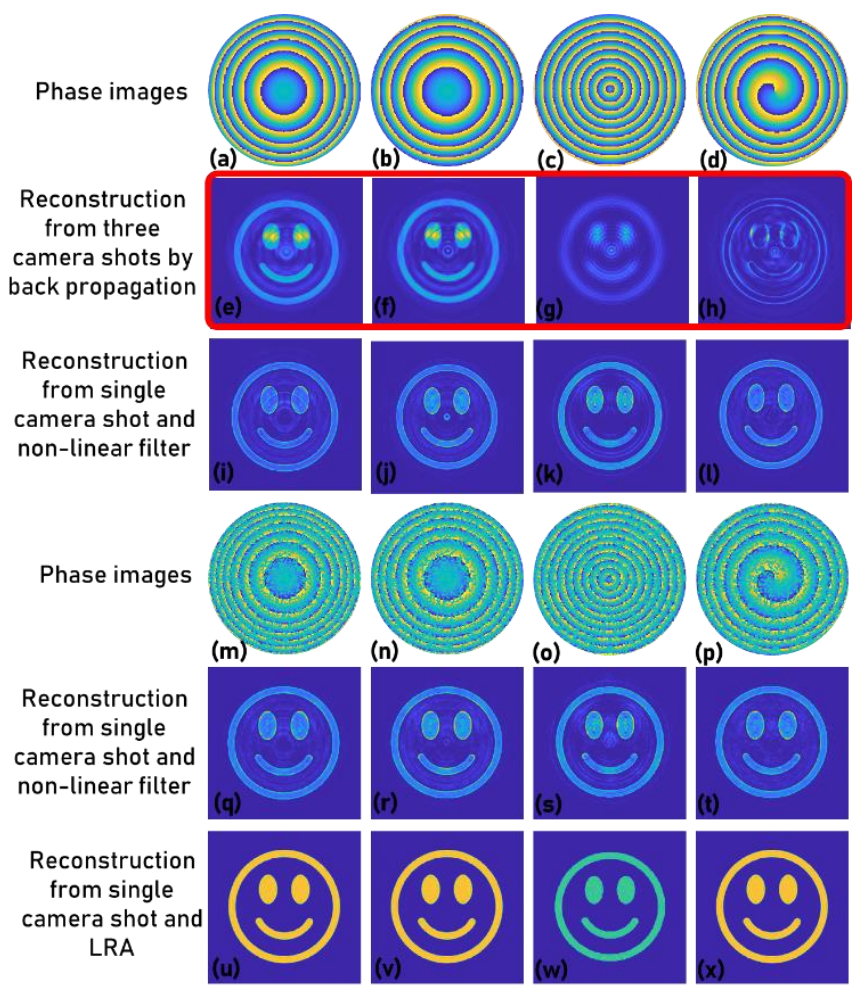

Fig. 2 Phase images of (a) QPM, (b) axilens, (c) axicon and (d) SQPM. Reconstruction results of FINCH in polarization multiplexing scheme with three camera shots and by back propagation for (e) QPM, (f) axilens, (g) axicon and (h) SQPM. Reconstruction results of FINCH in polarization multiplexing scheme with single camera shot and non-linear correlation (i) QPM, (j) axilens, (k) axicon and (l) SQPM. Phase images of randomly multiplexed constant matrix and (m) QPM, (n) axilens, (o) axicon and (p) SQPM. Reconstruction results of FINCH in spatial multiplexing scheme with non-linear correlation for (q) QPM, (r) axilens, (s) axicon and (t) SQPM. Reconstruction results of FINCH in spatial multiplexing scheme with single camera shot and LRA (u) QPM, (v) axilens, (w) axicon and (x) SQPM.

The reconstruction results by Fresnel propagation shown in Figs. 2(e)-2(h) shows that the characteristics of the modulation function has been faithfully transferred to the image characteristics. The axicon generated stronger side lobes and the SQPM generated edge-enhanced images of the object. The reconstruction results shown in Figs. 2(i)-(l) by cross-correlation by a non-linear filter shows only a weaker transfer of the characteristics. The results in Figs. 2(q)-2(x) shows a behavior which is nearly independent of the function of the phase mask.

The variation of the normalized intensity at the origin $(x=0, y=0)$ of the reconstructed point images with distance is plotted for the two cases of FINCH with QPM and axicon with Fresnel back propagation and non-linear correlation and compared with the direct image's intensity variations in Fig. 3 . It is seen from the Fig. 3 that the axial sensitivity of FINCH is lower than that of direct imaging and when an axicon is used instead of a QPM, the sensitivity decreases further. The appearance of peaks indicate the repetition of the pattern and the degree of pattern matching is exhibited by the value of the peak. The interesting point is that the behavior of FINCH with reconstruction by cross-correlation is not as expressive of the modulation function as the original version as the reconstructing function is dependent upon the modulation function of the phase mask. The results of axial correlations can be directly extended to spectral correlations based on the Fresnel propagator given as $e^{j \frac{\pi R^{2}}{\lambda z}}$ which controls the amplitude and phase within the paraxial regions. Any change in the distance $z$ can be compensated by an equal and opposite change in $\lambda$. Therefore, the intensity is expected to change by the same value when $\lambda$ varies by the same factor as $z$.

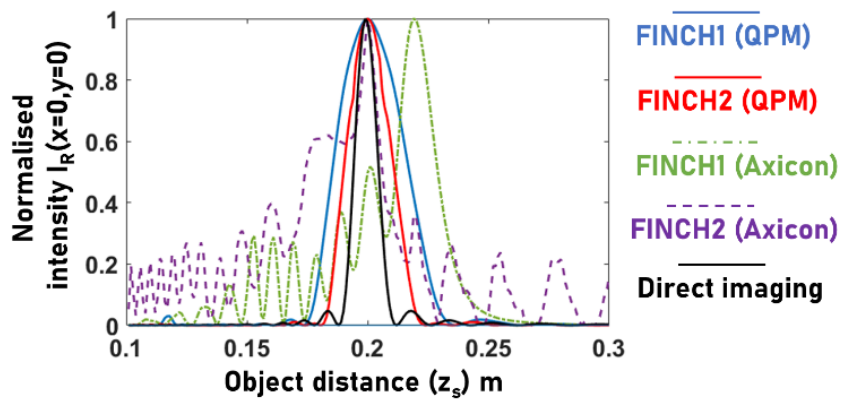

Fig. 3 Plot of $I(x=0, y=0)$ for FINCH (QPM), FINCH (axicon) and direct imaging for variation in the object distance $z_{S}(0.1$ to $0.3 \mathrm{~m})$ for FINCH1 - reconstruction by back propagation and FINCH2 reconstruction by cross-correlation.

The scattering ratio of the mask in the spatial multiplexing scheme is engineered using Gerchberg-Saxton algorithm (Fig. $4(\mathrm{a}))$ and three phase masks are synthesized with scattering ratio $\sigma=0.02,0.04,0.1$ and 0.5 as shown in Figs. 4(b)-4(d) respectively $[3,29]$. FINCH with a QPM and axicon were investigated for the above cases of scattering ratios.

The spatial multiplexing approach is studied next for $\sigma=0.02$, $0.04,0.1$ and 0.5 [29]. The plot of the normalized intensity at the origin $(x=0, y=0)$ of the reconstructed point images with distance is plotted for $\sigma=0.02,0.04,0.1$ and 0.5 as shown in Fig. 5. From Fig. 
there are two major observations. The expression of the modulation function in the imaging characteristics decreases with an increase in the scattering ratio $\sigma$ and in general, the imaging characteristics are substantially suppressed in spatial multiplexing method than in polarization multiplexing scheme. The mechanism of spatial multiplexing suppresses any modulation function and renders a uniform behavior but only dependent upon the scattering ratio, similar to that of coded aperture correlation holography [3, 30]. This has advantages as well as disadvantages. The advantage is that the system is expected to be insensitive to any aberrations and the disadvantage is that the system cannot transfer faithfully any characteristics from the modulating phase mask to imaging.
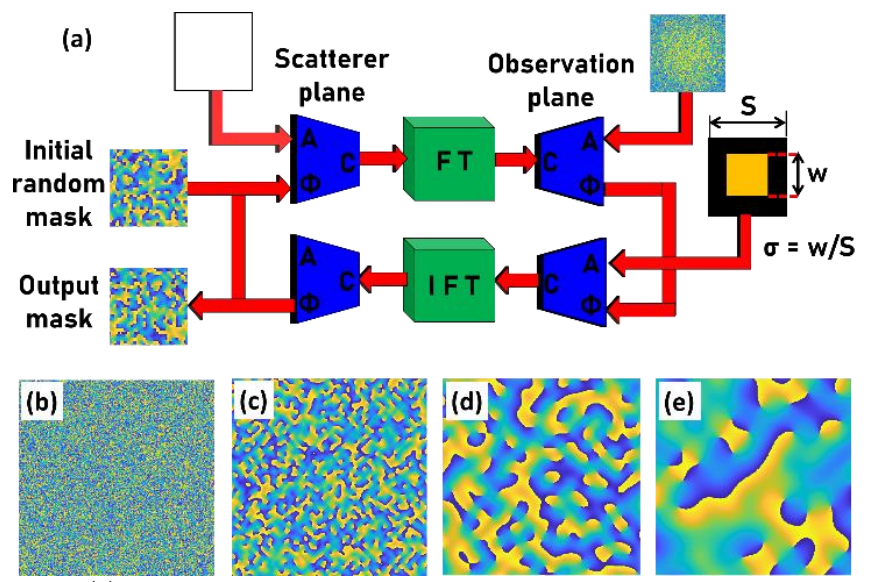

Fig. 4 (a) Gerchberg Saxton algorithm and generated phase masks with (b) $\sigma=0.5$, (c) $\sigma=0.1$ and (d) $\sigma=0.04$ and (e) $\sigma=0.02$.

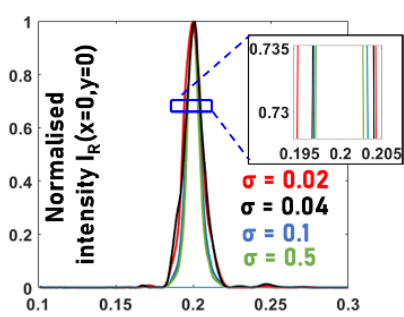

(a) Object distance $\left(z_{\mathrm{s}}\right) \mathrm{m}$

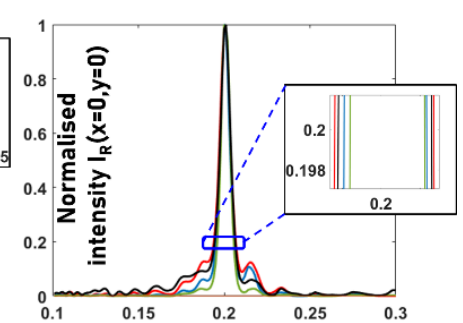

(b) Object distance $\left(z_{\mathrm{s}}\right) \mathrm{m}$
Fig. 5 Plot of $I(x=0, y=0)$ for (a) FINCH (QPM) and (b) FINCH (axicon) for variation in the object distance $z_{s}(0.1$ to $0.3 \mathrm{~m})$ for different scattering ratios $\sigma=0.02,0.04,0.1$ and 0.5 .

To experimentally analyse the spatial multiplexing system and to confirm the above observations, two cases are considered. In the two cases, FINCH is realised using randomly multiplexed $(\sigma=$ 0.04) QPMs and randomly multiplexed QPM and axicon. The two elements were fabricated using electron beam lithography (EBL:RAITH1502) for a central wavelength of $\lambda=617 \mathrm{~nm}$ and a diameter of $5 \mathrm{~mm}$. The QPMs were designed for $z_{S}=5 \mathrm{~cm}, z_{h}=10$ $\mathrm{cm}$ and the period of the axicon is $60 \mu \mathrm{m}$ in the second element. PMMA 950K (A7) resist was spin coated on Indium Tin Oxide (ITO) coated glass substrates and developed using Methyl IsoButyl Ketone (MIBK) and Iso Propyl Alcohol (IPA) solutions. A electron beam dose of $150 \mu \mathrm{C} / \mathrm{cm}^{2}$ was used and a writing time of 6 hours for each element. The optical microscope images of the diffractive elements are shown in Figs. 6(a) and 6(b), respectively. The holograms recorded for $\lambda=617 \mathrm{~nm}, z_{S}=5 \mathrm{~cm}$ to $6 \mathrm{~cm}$ in steps of $1 \mathrm{~mm}$ for the above two cases were cross-correlated with the hologram recorded at $z_{s}=5 \mathrm{~cm}$ using a non-linear filter $(\alpha=0$ and
$B=0.5)$ and the logarithm of the cross-correlation values (maximum value $=10000)$ are plotted as shown in Fig. 7 . The experiment is then repeated by replacing the source with $\lambda=530$ $\mathrm{nm}$. The cross-correlation values for $z_{S}=5 \mathrm{~cm}$ when switching between the two sources for two QPMs and QPM and axicon are $0.7512 \times 10^{-3}$ and $0.3947 \times 10^{-3}$ respectively. The plot in Fig. 7 shows similar behavior for QPM and axicon as the imaging characteristics transferred from the modulation function is suppressed by the spatial random multiplexing. The similar crosscorrelation values for QPM and axicon when the wavelength is varied indicates the same effect.

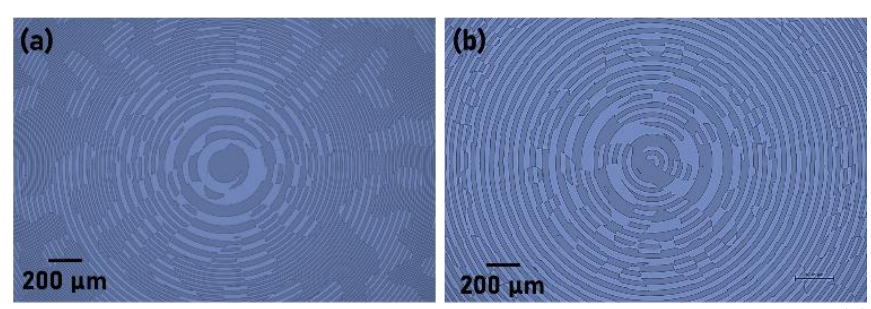

Fig. 6 Optical microscope images of randomly multiplexed (a) QPMs and (b) QPM and axicon.

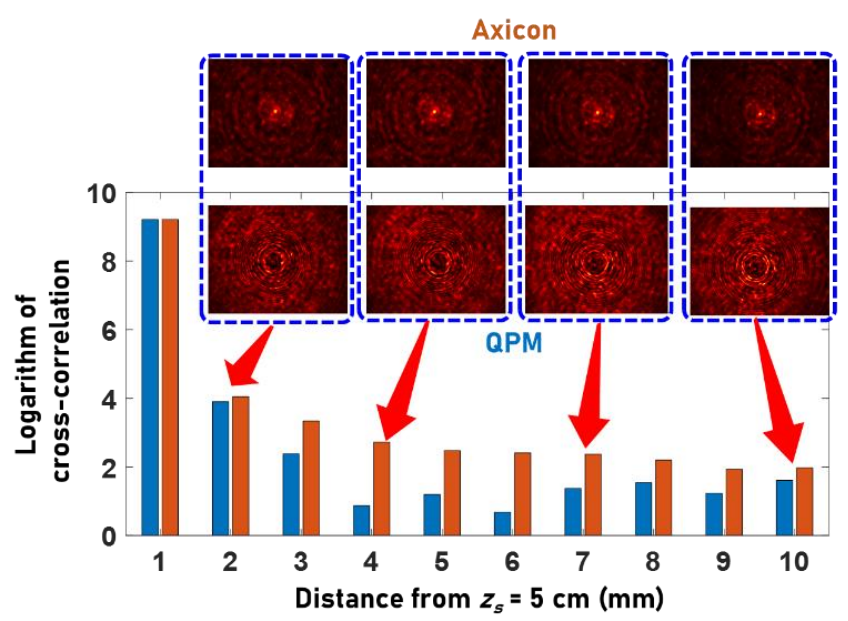

Fig. 7 Plot of the logarithm of the cross-correlation value for variation in distance along from $Z_{s}=5 \mathrm{~cm}$. The holograms recorded at $z_{S}=5.2 \mathrm{~cm}, 5.4 \mathrm{~cm}, 5.7 \mathrm{~cm}$ and $6 \mathrm{~cm}$ are shown.

FINCH is studied in different optical configurations using two reconstruction methods namely Fresnel back propagation and cross-correlation by a non-linear filter. It is observed that original FINCH in which a complex hologram is formed by the superposition of at least three phase-shifted holograms and reconstruction by back propagation faithfully expresses the characteristics of the modulation function in imaging. This is due to the condition that the reconstructing function is independent upon the modulation function. This is true for both spatial multiplexing as well as polarization multiplexing. FINCH in polarization multiplexing scheme and reconstruction by crosscorrelation could not express the characteristics of the modulation function accurately as the reconstructing function is dependent upon the modulation function. However, this method was able to express relative axial variations with respect to the reconstructing function. Therefore, the polarization multiplexing and reconstruction by cross-correlation can partially express the characteristics of the modulation function in the imaging characteristics. The final method involving spatial multiplexing 
and reconstruction by cross-correlation suppresses most of the effects from the modulation function. An insignificant variation of the axial characteristics with respect to the scattering degree of the spatial multiplexing was observed. As the scattering degree increases, the imaging characteristics approaches a uniform behavior almost independent of the modulation function. We believe that the new findings will guide the design of future FINCH imagers. The proposed techniques will extend the application of FINCH to single shot 3D colour imaging suitable for imaging focal spots in laser machining applications which are very bright and dynamic. In particular, the space-time evolution inside laser induced material breakdown which is used for X-ray and $\mathrm{THz}$ beam generation will be studied using the modified FINCH.

\section{References}

1. J. Rosen, and G. Brooker, "Digital spatially incoherent Fresnel holography," Opt. Lett. 32, 912 (2007).

2. J. Rosen, and G. Brooker, "Non-Scanning Motionless Fluorescence Three-Dimensional Holographic Microscopy," Nat. Photonics 2, 190 (2008).

3. J. Rosen, A. Vijayakumar, M. Kumar, M. R. Rai, R. Kelner, Y. Kashter, A. Bulbul, and S. Mukherjee, "Recent advances in self-interference incoherent digital holography," Adv. Opt. Photonics 11, 1 (2019).

4. J. Hong \& M.K. Kim, "Overview of techniques applicable to self-interference incoherent digital holography” J. Eur. Opt. Soc, Rapid Publ. 8, 13077 (2013).

5. Y. Wan, T. Man, F. Wu, M. K. Kim, and D. Wang, "Parallel phase-shifting self-interference digital holography with faithful reconstruction using compressive sensing," Opt. Lasers Eng. 86, 38 (2016).

6. T. C. Poon, J. P. Liu, Introduction to modern digital holography: with MATLAB (Cambridge University, 2014).

7. J. Rosen, and R. Kelner, "Modified Lagrange invariants and their role in determining transverse and axial imaging resolutions of self-interference incoherent holographic systems," Opt. Express 22, 29048 (2014).

8. J. Rosen, N. Siegel, and G. Brooker, "Theoretical and experimental demonstration of resolution beyond the Rayleigh limit by FINCH fluorescence microscopic imaging," Opt. Express 19, 26249 (2011).

9. P. Bouchal, J. Kapitán, R. Chmelík, and Z. Bouchal, "Point spread function and two-point resolution in Fresnel incoherent correlation holography," Opt. Express 19, 15603 (2011).

10. X. Lai, S. Zeng, X. Lv, J. Yuan, and L. Fu, "Violation of the Lagrange invariant in an optical imaging system," Opt. Lett. 38, 1896 (2013).

11. Y. Kashter, A. Vijayakumar, Y. Miyamoto and J. Rosen, "Enhanced super-resolution using Fresnel Incoherent Correlation Holography with structured illumination," Opt. Lett. 41, 1558 (2016).

12. Kashter, A. Vijayakumar, and J. Rosen, "Resolving images by blurring - a new superresolution method using a scattering mask between the observed objects and the hologram recorder," Optica 4, 932 (2017).

13. T. Tahara, T. Kanno, Y. Arai, and T. Ozawa, "Single-shot phase-shifting incoherent digital holography," J. Opt. 19, 065705 (2017).

14. T. Nobukawa, T. Muroi, Y. Katano, N. Kinoshita, and N. Ishii, "Single-shot phase-shifting incoherent digital holography with multiplexed checkerboard phase gratings," Opt. Lett. 43, 1698 (2018).
15. X. Quan, O. Matoba, and Y. Awatsuji, "Single-shot incoherent digital holography using a dual focusing lens with diffraction gratings," Opt. Lett. 42, 383 (2017).

16. J. Hong and M. K. Kim, "Single-shot self-interference incoherent digital holography using off-axis configuration," Opt. Lett. 38, 5196 (2013).

17. D. Liang, Q. Zhang, and J. Liu, "Single-shot Fresnel incoherent digital holography based on geometric phase lens," J. Mod. Opt. 67, 92 (2020).

18. J. Rosen, B. Katz, and G. Brooker, "Fresnel incoherent correlation hologram-a review." Chin. Opt. Lett. 7, 11341141 (2009).

19. A. Vijayakumar, T. Katkus, S. Lundgaard, D. Linklater, E. P. Ivanova, S. H. Ng, and S. Juodkazis, "Fresnel incoherent correlation holography with single camera shot." arXiv:1911.08291 (2019).

20. M. R. Rai, A. Vijayakumar and J. Rosen, "Non-linear Adaptive Three-Dimensional Imaging with interferenceless coded aperture correlation holography (I-COACH)” Opt. Express 26, 18143 (2018).

21. P. Bouchal and Z. Bouchal, "Selective edge enhancement in three-dimensional vortex imaging with incoherent light," Opt. Lett. 37, 2949 (2012).

22. V. Anand, T. Katkus, S. Juodkazis, "Randomly multiplexed diffractive lens and axicon for spatial and spectral imaging," Micromachines, 11, 437 (2020).

23. N. Davidson, A. A. Friesem, and E. Hasman, "Holographic axilens: high resolution and long focal depth," Opt. Lett. 16, 523 (1991).

24. B. S. Padilla, A. Žukauskas, A. Aleksanyan, A. Balčytis, M. Malinauskas, S. Juodkazis, and E. Brasselet, "Wrinkled axicons: shaping light from cusps," Opt. Express 24, 24075 (2016).

25. R. Dharmavarapu, S. Bhattacharya, and S. Juodkazis, "Diffractive optics for axial intensity shaping of bessel beams," J. Opt. 20, 085606 (2018).

26. A. Vijayakumar, C. Rosales-Guzmán, M. R. Rai, J. Rosen, O. V. Minin, I. V. Minin, and A. Forbes, "Generation of structured light by multilevel orbital angular momentum holograms," Opt. Express 27, 6459 (2019).

27. T. Xu, J. He, H. Ren, Z. Zhao, G. Ma, Q. Gong, S. Yang, L. Dong, and F. Ma, "Edge contrast enhancement of Fresnel incoherent correlation holography (FINCH) microscopy by spatial light modulator aided spiral phase modulation," Opt. Express 25, 29207 (2017).

28. D. A. Fish, A. M. Brinicombe, E. R. Pike, and J. G. Walker, "Blind deconvolution by means of the Richardson-Lucy algorithm," J. Opt. Soc. Am. A 12, 58-65 (1995).

29. S. J. Lathika, V. Anand and S. Bhattacharya, "A compact single channel interferometer to study vortex beam propagation through scattering layers," Sci. Rep. 10, 296 (2020).

30. A. Vijayakumar and J. Rosen, "Interferenceless coded aperture correlation holography - a new technique for recording incoherent digital holograms without two-wave interference," Opt. Express 25, 13883 (2017).

Funding Sources. NATO grant No. SPS-985048, Linkage grant LP190100505 and the Australian Research Council Discovery grant DP190103284 are acknowledged for funding. This work was performed in part at the Swinburne's Nanofabrication Facility (Nanolab). 
Acknowledgements. VA thanks Prof. Joseph Rosen, Ben Gurion University, Israel, for the useful discussions. 